\title{
On the price of anarchy and stability of correlated equilibria of linear congestion games*
}

\author{
George Christodoulou Elias Koutsoupias ${ }^{\dagger}$
}

February 14, 2005

\begin{abstract}
We consider the price of stability for Nash and correlated equilibria of linear congestion games. The price of stability is the optimistic price of anarchy, the ratio of the cost of the best Nash or correlated equilibrium over the social optimum. We show that for the sum social cost, which corresponds to the average cost of the players, every linear congestion game has Nash and correlated price of stability at most 1.6. We also give an almost matching lower bound of $1+\sqrt{3} / 3=1.577$.

We also consider the price of anarchy of correlated equilibria. We extend some of the results in $[2,4]$ to correlated equilibria and show that for the sum social cost, the price of anarchy is 2.5. The same bound holds for symmetric games as well. This matches the lower bounds given in [2, 4] for pure Nash equilibria. We also extend the results in [2] for weighted congestion games to correlated equilibria. Specifically, we show that when the social cost is the total latency, the price of anarchy is $(3+\sqrt{5}) / 2=2.618$.
\end{abstract}

\section{Introduction}

Recently, a new vigorous subfield of computer science emerged which studies how the viewpoint and behavior of users affects the performance of computer networks or systems, by modeling the situation as a game.

One of the most important questions in game theory is what is the correct solution concept of a game. There are many proposals but three types of equilibria stand out in the literature of non-cooperative game theory. The first and stronger equilibrium occurs when there are dominant strategies, in which each player has an optimal strategy independently of what the other players do. Unfortunately not every game has such a solution (and sometimes even if it has one, as in the case of the game of prisoner's dilemma, it may lead to unexpected solutions). The second and the most well-known equilibrium is the Nash equilibrium, whose existence is assured for every finite game by the famous theorem by Nash. The third type of equilibria is the notion of correlated equilibrium, introduced by Aumann [1], and which recently is the focus of computational studies (see for example [22, 21]).

A correlated strategy for a game is a probability distribution over all the possible pure strategy profiles. One can interpret the situation as follows: there is a trusted mediator who performs the random experiment and announces the resulted strategies to each player in private. The players although they know the distribution, they are not informed about the outcome of the experiment but just about their own strategy. They may choose to follow or not the mediator's advice according to their utility function. A correlated strategy is a correlated equilibrium if no player has any reason to unilaterally disobey to the mediator's advice. Roughly speaking this paradigm is also an interpretion for mixed Nash equilibria with the exception that

\footnotetext{
${ }^{*}$ Research supported in part by the IST (FLAGS, IST-2001-33116) program.

${ }^{\dagger}$ University of Athens. \{gchristo,elias\}@di.uoa.gr
} 
the distribution is formed by independent random experiments (one for each player) over the possible pure strategies for each player.

The three types of equilibria (dominant, Nash, and correlated) are related by inclusion: the set of Nash equilibria contains the dominant strategies and the set of correlated equilibria contains all Nash equilibria. Thus the existence of correlated equilibria is guaranteed for every finite game.

One of the main tools in the study of selfish behavior is the price of anarchy [14, 20], a measure that compares the worst case performance Nash equilibrium to that of the optimal allocation. Naturally, the concept of the price of anarchy extends to correlated equilibria. If we know only that the players play at some equilibrium, the price of anarchy bounds the deterioration of system performance due to selfish behavior. On the other hand, there is the optimistic point of view in which the players are guided to play at the best Nash equilibrium. Especially with correlated equilibria, the latter makes much more sense: The mediator who selects the probability distribution, the correlated equilibrium, and presents it to the players, can select the correlated equilibrium with minimum system cost. In other words, one can view correlated equilibria as a mechanism for enforcing good behavior on selfish users. The optimistic price of anarchy of the best equilibrium is also called price of stability [3].

In this paper we consider the price of anarchy and stability of Nash and correlated equilibria of congestion games, a class of games which suitably models traffic networks and abstracts well many game-theoretic situations in networks and systems. Congestion games, introduced by Rosenthal [23], provide a common framework for almost all previous studies of price of anarchy which originated in [14] and [26]. Congestion games have the fundamental property that a pure Nash equilibrium always exists. In [18], it is proved that congestion games (also called potential games) are characterized by a potential: the local optima of the potential correspond exactly to mixed Nash equilibria (see [8] for computational issues related to this issue).

Whether one studies the price of anarchy or the price of stability, a critical matter is the definition of the social optimum: the system allocation mostly desired by the system designer. From the system designers point of view there are two natural notions of social cost: the maximum or the average (or sum) cost among the players. For the original model of parallel links in [14], the social cost was the maximum cost among the players. For the Wardrop model studied by Roughgarden and Tardos [26], the social cost was the average player cost. A third social cost is the total latency which is the sum of squares of the loads on each facility. For unweighted games this is identical to the average (or sum) cost among the players.

Here we deal with the average social cost but we also consider the maximum social cost and the total latency social cost (for weighted congestion games). We also consider the price of anarchy of the natural subclass of symmetric congestion games, where the available actions are the same for all the players.

\subsection{Our results}

We study linear general - not only network - congestion games with cost (latency) functions of the form $f_{e}(k)=a_{e} k+b_{e}$ with nonnegative coefficients. We focus mainly on the sum social cost which is the sum of the cost of all players and we consider all three types of equilibria: dominant strategies, pure and mixed Nash equilibria, and correlated equilibria.

Price of stability: For linear congestion games we give an upper bound of 1.6 for Nash and correlated equilibria (Theorem 1). Although bounding directly the price of stability seems hard - after all, we need to bound the best not the worst equilibrium - we resort to a clever trick by making use of the potential of congestion games. More specifically, instead of bounding the cost of the best equilibrium, we bound the cost of the pure Nash equilibrium which has minimum potential. Since every local optimum of the potential corresponds to a pure Nash 
equilibrium, such a Nash equilibrium is guaranteed to exist. In fact, the proof of Theorem 1 does not even need to consider the Nash equilibrium with minimum potential. All we need to consider is that the potential of the Nash equilibrium is less than the potential of the optimal strategies.

We give a non-trivial lower bound of $1+\frac{\sqrt{3}}{3} \approx 1.577$ for dominant strategies (Theorem 2 ). This is perhaps the most technical part of this work. Naturally, both the upper and lower bounds hold for all three types of equilibria (dominant strategies, Nash, and correlated equilibria). An open problem is to close the little gap between 1.577 and 1.6.

We also observe that for the max social cost (i.e., the maximum cost among the players) the price of stability is $\Theta(\sqrt{N})$ (Theorem 3). This follows by a minor modification to the lower bound (about pure Nash equilibria) given in [4].

Price of anarchy: For linear congestion games, we extend some of the results of our forthcoming STOC'05 paper [4] on the price of anarchy of Nash equilibria to correlated equilibria and at the same time improve them. More specifically, we show that the correlated price of anarchy of the sum social cost is 2.5 for the asymmetric case (Theorem 4) and $\frac{5 N-2}{2 N+1}$ for the symmetric case (Theorem 5), where $N$ is the number of players. Since in [4], we had matching lower bounds for pure Nash equilibria, these are also tight bounds for Nash equilibria.

We also extend the results of [2] about the price of anarchy of Nash equilibria for weighted linear congestion games when the social cost is the total latency: The price of anarchy of correlated equilibria is $\frac{3+\sqrt{5}}{2} \approx 2.618$ (Theorem 6 ). Although we prove a more general result, our proof is substantially simpler.

\subsection{Related work}

The closer works in spirit, results, and techniques are the STOC'05 papers [4, 2]. Both these papers study the price of anarchy of pure and mixed Nash equilibria for linear congestion games and congestion games with polynomial delay functions. In particular, both papers show that price of anarchy of pure Nash equilibria of linear congestion games is 2.5 for the average social cost (and the total latency cost which is identical in this case). Paper [4] also shows that the same bound holds also for symmetric games for both the average and the maximum social cost but it gets up to $\Theta(\sqrt{N})$ for the maximum social cost (in the general case). It also gives a 2.618 bound for mixed equilibria and the average social cost. On the other hand, [2] considers weighted congestion games for the total latency social cost and shows that the price of anarchy for both pure and mixed equilibria is 2.618 .

The study of the price of anarchy was initiated in [14], for (weighted) congestion games of $m$ parallel links. The price of anarchy for the maximum social cost is proved to be $\Omega\left(\frac{\log m}{\log \log m}\right)$, while in $[16,13,7]$ they proved $\Theta\left(\frac{\log m}{\log \log m}\right)$. In [7] extended the result to $m$ parallel links with different speeds and showed that the price of anarchy is $\Theta\left(\frac{\log m}{\log \log \log m}\right)$. In [6], more general latency functions are studied, especially in relation to queuing theory. For the same model of parallel links, [10] and [15] consider the price of anarchy for other social costs.

In [28], the special case of congestion games in which each strategy is a singleton set is considered. They give bounds for the case of the average social cost. For the same class of congestion games and the maximum social cost, [11] showed that the price of anarchy is $\Theta(\log N / \log \log N)$ (a similar, perhaps unpublished, result was obtained by the group of [28]). The case of singleton strategies is also considered in [12] and [15].

In [9], they consider the mixed price of anarchy of symmetric network weighted congestion games, when the network is layered.

The non-atomic case of congestion games was considered in [26, 27] where they showed that for linear latencies the average price of anarchy is $4 / 3$. They also extended this result to polynomial latencies. Furthermore, $[5,25]$ considered the social cost of maximum latency. 


\section{The model}

A congestion game is a tuple $\left(N, E,\left(\mathcal{S}_{i}\right)_{i \in N},\left(f_{e}\right)_{e \in E}\right)$ where $N=\{1, \ldots, n\}$ is the set of players, $E$ is a set of facilities, $\mathcal{S}_{i} \subseteq 2^{E}$ is a collection of pure strategies for player $i$ : a pure strategy $A_{i} \in \mathcal{S}_{i}$ is a set of facilities, and finally $f_{e}$ is a cost (or latency) function associated with facility $j$. We are concerned with linear cost functions: $f_{e}(k)=a_{e} \cdot k+b_{e}$ for nonnegative constants $a_{e}$ and $b_{e}$.

A pure strategy profile $A=\left(A_{1}, \ldots, A_{n}\right)$ is a vector of strategies, one for each player. The cost of player $i$ for the pure strategy profile $A$ is given by $c_{i}(A)=\sum_{e \in A_{i}} f_{e}\left(n_{e}(A)\right)$, where $n_{e}(A)$ is the number of the players using $e$ in $A$. A pure strategy profile $A$ is a Nash equilibrium if no player has any reason to unilaterally deviate to another pure strategy: $\forall i \in N, \forall s \in \mathcal{S}_{i} \quad c_{i}(A) \leq$ $c_{i}\left(A_{-i}, s\right)$, where $\left(A_{-i}, s\right)$ is the strategy profile produced if just player $i$ deviates from $A_{i}$ to $s$.

The social cost of $A$ is either the maximum cost of a player $\operatorname{MAx}(A)=\max _{i \in N} c_{i}(A)$ or the average of the players' costs. For simplicity, we consider the sum of all costs (which is $N$ times the average cost) $\operatorname{Sum}(A)=\sum_{i \in N} c_{i}(A)$. These definitions extend naturally to the cases of mixed and correlated strategies (with expected costs, of course).

A mixed strategy $p_{i}$ for a player $i$, is a probability distribution over his pure strategy set $\mathcal{S}_{i}$. A correlated strategy $q$ for a set of players is any probability distribution over the set of possible combinations of pure strategies that these players can choose. That is a correlated strategy for $N$ is any probability distribution in $\Delta(\mathcal{S})$, where $\mathcal{S}=\times_{i \in N} \mathcal{S}_{i}$.

Given a correlated strategy $q$ in $\Delta(\mathcal{S})$, the expected cost of a player $i \in N$ is

$$
c_{i}(q)=\sum_{s \in \mathcal{S}} q(s) c_{i}(s)
$$

A correlated strategy $q \in \Delta(\mathcal{S})$ is a correlated equilibrium if $q$ satisfies the following condition

$$
c_{i}(q) \leq \sum_{s \in \mathcal{S}} q(s) c_{i}\left(s_{-i}, \delta_{i}\left(s_{i}\right)\right), \quad \forall i \in N, \quad \forall \delta_{i}\left(s_{i}\right): \mathcal{S}_{i} \rightarrow \mathcal{S}_{i}
$$

A congestion game is symmetric (or single-commodity) if all the players have the same strategy set: $\mathcal{S}_{i}=\mathcal{C}$. We use the term "asymmetric" (or multi-commodity) to refer to all games (including the symmetric ones).

The correlated price of anarchy of a game is the worst-case ratio, among all correlated equilibria, of the social cost over the optimum social cost, opt $=\min _{P \in \mathcal{S}} s c(P)$.

$$
P A=\sup _{q \text { is a corr. eq. }} \frac{s c(q)}{o p t}
$$

The correlated price of stability of a game is the best-case ratio, among all correlated equilibria, of the social cost over the optimum.

$$
P S=\inf _{q \text { is a corr. eq. }} \frac{s c(q)}{o p t}
$$

When we refer to the price of stability (resp. anarchy) of a class of games, we mean the maximum (or supremum) price of stability (resp. anarchy) among all games in the class.

In weighted congestion games, each player controls an amount of traffic $w_{i}$, and the cost of a facility $e$ depends on the total load on the facility. For this case, some of our results involve the total latency social cost. For a pure strategy profile $A \in \mathcal{S}$, the total latency is defined as $C(A)=\sum_{e \in E} \theta_{e}(A) \cdot f_{e}\left(\theta_{e}(A)\right.$. Notice that the sum and the total latency social costs coincide for unweighted congestion games. 


\section{The correlated price of stability of congestion games}

In this section we study the price of stability of linear congestion games. We will use the following simple lemma:

Lemma 1. For every nonnegative integers $\alpha, \beta$ :

$$
\alpha \beta+2 \beta-\alpha \leq \frac{1}{8} a^{2}+2 \beta^{2}
$$

Proof.

$$
2 \beta^{2}+\frac{1}{8} a^{2}-\alpha \beta-2 \beta+a=(\alpha-4(\beta-1))^{2}+16(\beta-1) \geq 0
$$

Define the potential of a strategy profile $A$ of a congestion game to be $P(A)=\sum_{e \in E} \sum_{i=1}^{n_{e}(A)} f_{e}(i)$. The potential has the nice property that when $P(A)$ is a local optimum, then $A$ is a pure Nash equilibrium. To establish an upper bound on the price of stability, we simply bound the price of anarchy of the subclass of Nash equilibria whose potential does not exceed the potential of the optimal allocation. Clearly, by the property of the potential, this subclass of Nash equilibria is not empty.

Theorem 1. Let $A$ be a pure Nash equilibrium and $P$ be any pure strategy profile such that $P(A) \leq P(P)$, then $\operatorname{Sum}(A) \leq \frac{8}{5} \operatorname{Sum}(P)$. This shows that the price of stability of correlated equilibria is at most 1.6.

Proof. From the potential inequality we have

$$
\begin{array}{r}
P(A)=\sum_{e \in E} \sum_{i=1}^{n_{e}(A)} f_{e}(i)=\frac{1}{2} \operatorname{Sum}(A)+\frac{1}{2} \sum_{e \in E}\left(a_{e}+b_{e}\right) n_{e}(A) \leq \\
P(P)=\frac{1}{2} \operatorname{Sum}(P)+\frac{1}{2} \sum_{e \in E}\left(a_{e}+b_{e}\right) n_{e}(P)
\end{array}
$$

From the Nash inequality we have

$$
\operatorname{Sum}(A) \leq \sum_{e \in E} n_{e}(P) f_{e}\left(n_{e}(A)+1\right)=\sum_{e \in E} a_{e} n_{e}(A) n_{e}(P)+\sum_{e \in E}\left(a_{e}+b_{e}\right) n_{e}(P) .
$$

So if we sum the above inequalities and use Lemma 1 we get

$$
\begin{array}{r}
2 \operatorname{Sum}(A) \leq \sum_{e \in E} a_{e}\left(n_{e}(A) n_{e}(P)+n_{e}^{2}(P)+2 n_{e}(P)-n_{e}(A)\right)+\sum_{e \in E} b_{e}\left(3 n_{e}(P)-n_{e}(A)\right) \leq \\
\frac{1}{8} \operatorname{Sum}(A)+3 \operatorname{Sum}(P)
\end{array}
$$

and the lemma follows.

\subsection{Lower bound}

We now provide an almost matching lower bound.

Theorem 2. There are linear congestion games whose dominant equilibrium - and therefore the Nash and correlated equilibria - have price of stability of the SUM social cost approaching $1+\sqrt{3} / 3 \approx 1.577$ as the number of players $N$ tends to infinity. 
Proof. We describe a game of $N$ players with parameters $\alpha, \beta$, and $m$ which we will fix later to obtain the desired properties. Each player $i$ has two strategies $A_{i}$ and $P_{i}$, where the strategy profile $\left(A_{1}, \ldots, A_{N}\right)$ will be the equilibrium and $\left(P_{1}, \ldots, P_{N}\right)$ will have optimal social cost.

There are 3 types of facilities:

- $N$ facilities $\alpha_{i}, i=1, \ldots, N$, each with cost function $f(k)=\alpha k$. Facility $\alpha_{i}$ belongs only to strategy $P_{i}$.

- $N(N-1)$ facilities $\beta_{i j}, i, j=1, \ldots, N$ and $i \neq j$, each with cost $f(k)=\beta k$. Facility $\beta_{i j}$ belongs only to strategies $A_{i}$ and $P_{j}$.

- $\left(\begin{array}{l}N \\ m\end{array}\right)$ facilities $\gamma_{S}$, one for each subset $S$ of $\{1, \ldots, N\}$ of cardinality $m$ and with cost function $f(k)=k$. Facility $\gamma_{S}$ belongs to strategy $A_{i}$ iff $i \notin S$ and to strategy $P_{j}$ iff $j \in S$.

We will first compute the cost of every player and every strategy profile. By symmetry, we need only to consider the cost $\operatorname{cost}_{A}(k)$ of player 1 and the cost $\operatorname{cost}_{P}(k)$ of player $N$ of the strategy profile $\left(A_{1}, \ldots, A_{k}, P_{k+1}, \ldots, P_{N}\right)$. We could count the cost that every facility contributes to $\operatorname{cost}_{A}(k)$ and $\operatorname{cost}_{P}(k)$, but this results in complicated sums. A simpler way is to resort to probabilities and consider the contribution of a random facility of each of the 3 types. For example, consider a random facility $\gamma_{S}$ which is used by player 1 , i.e. $1 \notin S$. The probability that player $j=k+1, \ldots, N$ uses this facility is equal to the probability that $j \in S$ which is equal to $m /(N-1)$. Also the probability that player $i=2, \ldots, k$ uses the facility is equal to the probability that $i \notin S$ which is equal to $(N-1-m) /(N-1)$. Therefore the expected number of players that use the facility $\gamma_{S}$ is

$$
1+(N-k) \frac{m}{N-1}+(k-1) \frac{N-1-m}{N-1}=\frac{k N+m N-2 k m+m-k}{N-1} .
$$

Taking into account that there are $\left(\begin{array}{c}N-1 \\ m\end{array}\right)$ such facilities, the contribution of type 3 facilities to the cost $\operatorname{cost}_{A}(k)$ of player 1 is $\left(\begin{array}{c}N-1 \\ m\end{array}\right) \frac{k N+m N-2 k m+m-k}{N-1}$. With similar but simpler considerations we compute the contribution to $\operatorname{cost}_{A}(k)$ of facilities of the second type to be $(2 N-k-1) \beta$. Therefore,

$$
\operatorname{cost}_{A}(k)=(2 N-k-1) \beta+\left(\begin{array}{c}
N-1 \\
m
\end{array}\right) \frac{k N+m N-2 k m+m-k}{N-1} .
$$

Similarly, we compute

$$
\operatorname{cost}_{P}(k)=\alpha+(N+k-1) \beta+\left(\begin{array}{c}
N-1 \\
N-m
\end{array}\right) \frac{k N+m N-2 k m-m+k}{N-1} .
$$

(In fact by symmetry, and with the exception of the term $\alpha$, the cost $\operatorname{cost}_{P}(k)$ results from $\operatorname{cost}_{A}(k)$ when we replace $k$ and $m$ by $N-k$ and $N-m$, respectively.)

We now want to select the parameters $\alpha$ and $\beta$ so that the strategy profile $\left(A_{1}, \ldots, A_{N}\right)$ is dominant. Equivalently, at every strategy profile $\left(A_{1}, \ldots, A_{k}, P_{k+1}, \ldots, P_{N}\right)$, player $i, i=$ $1, \ldots, k$, has no reason to switch to strategy $P_{i}$. This is expressed by the constraint

$$
\operatorname{cost}_{A}(k) \leq \operatorname{cost}_{P}(k-1), \quad \text { for every } k=1, \ldots, N .
$$

Magically, all these constraints are satisfied by equality when

$$
\alpha=\left(\begin{array}{l}
N \\
m
\end{array}\right) \frac{N^{2}-2 m-2 N m+N}{2 N},
$$

and

$$
\beta=\left(\begin{array}{l}
N \\
m
\end{array}\right) \frac{N^{2}+4 m^{2}-4 N m-N}{2 N(N-1)} .
$$


(The mystery disappears when we observe that both $\operatorname{cost}_{A}(k)$ and $\operatorname{cost}_{P}(k)$ are linear in $k$.)

In summary, for the above values of the parameters $\alpha$ and $\beta$, we obtain the desired property that the strategy profile $\left(A_{1}, \ldots, A_{N}\right)$ is a dominant strategy. If we increase $\alpha$ by any small positive $\epsilon$, inequality (1) becomes strict and the dominant strategy is unique (and therefore unique Nash and correlated equilibrium).

We now want to select the value of the parameter $m$ so that the price of anarchy of this equilibrium is as high as possible. The price of anarchy is $\operatorname{cost}_{A}(N) / \operatorname{cost}_{P}(0)$ which for the above values of $\alpha$ and $\beta$ can be simplified to

$$
p a=\frac{3 N^{2}+6 m^{2}-8 N m-N}{2 N^{2}+6 m^{2}-6 N m-2 m} .
$$

For $m / N \approx 1 / 2-\sqrt{3} / 6$, the price of anarchy tends to $p a=1+\sqrt{3} / 3 \approx 1.577$, as $N$ tends to infinity.

Theorem 3. The price of stability for dominant strategies for asymmetric congestion games is $\Theta(\sqrt{N})$.

Proof. A minor modification of the example in the lower bound in [4] works. We simply add a small term $\epsilon$ to the bad Nash equilibrium case, in order to turn the strategies into dominant ones. On the other hand, the price of stability cannot be more that the price of anarchy of pure Nash equilibria which shown in [4] to be $O(\sqrt{N})$.

\section{The correlated price of anarchy of congestion games}

We now turn our attention to the correlated price of anarchy of congestion games for linear facility delay functions and for the average social cost.

The following is a simple fact which will be useful in the proof of the next theorem.

Lemma 2. For every pair of nonnegative integers $\alpha, \beta$, it holds

$$
\alpha(\beta+1) \leq \frac{1}{3} \alpha^{2}+\frac{5}{3} \beta^{2} .
$$

Theorem 4. The correlated price of anarchy of the average social cost is $\frac{5}{2}$.

Proof. The lower bound is established in $[2,4]$ (for pure equilibria). To establish the upper bound, let $q$ be a correlated equilibrium and $P$ be an optimal (or any other) allocation. The cost of player $i$ at the correlated equilibrium is $c_{i}(q)=\sum_{s \in \mathcal{S}} q(s) c_{i}(s)=\sum_{s \in \mathcal{S}} q(s) \sum_{e \in s_{i}} f_{e}\left(n_{e}(s)\right)$. We want to bound the expected social cost, the sum of the expected costs of the players: $\operatorname{Sum}(q)=\sum_{i} c_{i}(q)=\sum_{s \in \mathcal{S}} q(s) \sum_{e \in E} n_{e}(s) f_{e}\left(n_{e}(s)\right)$, with respect to the optimal cost $\operatorname{Sum}(P)=$ $\sum_{i} c_{i}(P)=\sum_{e \in E} n_{e}(P) f_{e}\left(n_{e}(P)\right)$.

At the correlated equilibrium

$$
c_{i}(q)=\sum_{s \in \mathcal{S}} q(s) \sum_{e \in s_{i}} f_{e}\left(n_{e}(s)\right) \leq \sum_{s \in \mathcal{S}} q(s) \sum_{e \in P_{i}} f_{e}\left(n_{e}\left(s_{-i}, P_{i}\right)\right) \leq \sum_{s \in \mathcal{S}} q(s) \sum_{e \in P_{i}} f_{e}\left(n_{e}(s)+1\right)
$$

where $\left(s_{-i}, P_{i}\right)$ is the usual notation in Game Theory to denote the allocation that results when we replace $s_{i}$ by $P_{i}$. 
If we sum over all players $i$, we can bound the expected social cost as

$$
\begin{array}{r}
\operatorname{Sum}(q)=\sum_{i \in N} c_{i}(q) \leq \sum_{i \in N} \sum_{s \in \mathcal{S}} q(s) \sum_{e \in P_{i}} f_{e}\left(n_{e}(s)+1\right)= \\
\sum_{s \in \mathcal{S}} q(s) \sum_{e \in E} n_{e}(P) f_{e}\left(n_{e}(s)+1\right)= \\
\sum_{s \in \mathcal{S}} q(s) \sum_{e \in E} n_{e}(P)\left(a_{e}\left(n_{e}(s)+1\right)+b_{e}\right)= \\
\sum_{s \in \mathcal{S}} q(s) \sum_{e \in E}\left(a_{e}\left(n_{e}(P)\left(n_{e}(s)+1\right)+b_{e} n_{e}(P)\right) \leq\right. \\
\sum_{s \in \mathcal{S}} q(s) \sum_{e \in E}\left(a_{e}\left(\frac{1}{3} n_{e}^{2}(s)+\frac{5}{3} n_{e}^{2}(P)\right)+b_{e} n_{e}(P)\right) \leq \\
\sum_{s \in \mathcal{S}} q(s) \sum_{e \in E} \frac{1}{3}\left(a_{e} n_{e}^{2}(s)+b_{e}\left(n_{e}(s)\right)+\sum_{s \in \mathcal{S}} q(s) \sum_{e \in E} \frac{5}{3}\left(a_{e} n_{e}^{2}(P)+b_{e} n_{e}(P)\right)=\right. \\
\frac{1}{3} \operatorname{Sum}(q)+\frac{5}{3} \operatorname{SUM}(P),
\end{array}
$$

where the first inequality follows from Lemma 3 and the other one by the fact that $b_{e}$ is nonnegative.

For the symmetric games the correlated price of anarchy is also 5/2. In fact, as the next theorem establishes, it is slightly less: $\frac{5 N-2}{2 N+1}$. This is tight, as a matching lower bound for pure Nash equilibria in [4] shows.

Theorem 5. The average correlated price of anarchy of symmetric congestion games with linear cost functions is $\frac{5 N-2}{2 N+1}$.

Proof. For each player $i$ we have from the Nash inequalities that

$$
\begin{gathered}
c_{i}(q) \leq \sum_{s \in \mathcal{S}} q(s)\left(\sum_{e \in P_{1} \cap s_{i}} f_{e}\left(n_{e}(s)\right)+\sum_{e \in P_{1}-s_{i}} f_{e}\left(n_{e}(s)+1\right)\right)=\sum_{s \in \mathcal{S}} q(s)\left(\sum_{e \in P_{1}} f_{e}\left(n_{e}(s)+1\right)-\sum_{e \in P_{1} \cap s_{i}} a_{e}\right) \\
c_{i}(q) \leq \sum_{s \in \mathcal{S}} q(s)\left(\sum_{e \in P_{2} \cap s_{i}} f_{e}\left(n_{e}(s)\right)+\sum_{e \in P_{2}-s_{i}} f_{e}\left(n_{e}(s)+1\right)\right)=\sum_{s \in \mathcal{S}} q(s)\left(\sum_{e \in P_{2}} f_{e}\left(n_{e}(s)+1\right)-\sum_{e \in P_{2} \cap s_{i}} a_{e}\right) \\
c_{i}(q) \leq \sum_{s \in \mathcal{S}} q(s)\left(\sum_{e \in P_{N} \cap s_{i}} f_{e}\left(n_{e}(s)\right)+\sum_{e \in P_{N}-s_{i}} f_{e}\left(n_{e}(s)+1\right)\right)=\sum_{s \in \mathcal{S}} q(s)\left(\sum_{e \in P_{N}} f_{e}\left(n_{e}(s)+1\right)-\sum_{e \in P_{N} \cap s_{i}} a_{e}\right) .
\end{gathered}
$$

So,

$$
N \cdot c_{i}(q) \leq \sum_{s \in S} q(s)\left(\sum_{e \in E} n_{e}(P) f_{e}\left(n_{e}(s)+1\right)-\sum_{e \in s_{i}} a_{e} n_{e}(P)\right)
$$


If we sum over all $i \in N$ we get

$$
\begin{array}{r}
N \operatorname{Sum}(q) \leq \sum_{s \in S} q(s)\left(N \sum_{e \in E} n_{e}(P) f_{e}\left(n_{e}(s)+1\right)-\sum_{e \in E} a_{e} n_{e}(P) n_{e}(s)\right)= \\
\sum_{s \in S} q(s)\left(N \sum_{e \in E} n_{e}(P)\left(a_{e}\left(n_{e}(s)+1\right)+b_{e}\right)-\sum_{e \in E} a_{e} n_{e}(P) n_{e}(s)\right)= \\
\sum_{s \in S} q(s)\left((N-1) \sum_{e \in E} a_{e} n_{e}(P) n_{e}(s)+N \sum_{e \in E} a_{e} n_{e}(P)+N \sum_{e \in E} b_{e} n_{e}(P)\right)= \\
\sum_{s \in S} q(s)\left((N-1) \sum_{e \in E} a_{e} n_{e}(P)\left(n_{e}(s)+1\right)+\sum_{e \in E} a_{e} n_{e}(P)+N \sum_{e \in E} b_{e} n_{e}(P)\right) \leq \\
\sum_{s \in S} q(s)\left((N-1) \sum_{e \in E} a_{e}\left(\frac{1}{3} n_{e}^{2}(s)+\frac{5}{3} n_{e}^{2}(P)\right)+\sum_{e \in E} a_{e} n_{e}(P)+N \sum_{e \in E} b_{e} n_{e}(P)\right) \\
\sum_{s \in S} q(s)\left(\frac{N-1}{3} \operatorname{Sum}(s)+\frac{5 N-2}{3} \operatorname{Sum}(P)-\frac{2}{3}(N-1) \sum_{e \in E} b_{e} n_{e}(P)\right) \leq \\
\sum_{s \in S} q(s)\left(\frac{N-1}{3} \operatorname{Sum}(s)+\frac{5 N-2}{3} \operatorname{Sum}(P)\right)
\end{array}
$$

and the theorem follows.

\subsection{Asymmetric weighted games}

In this subsection we assume that the social cost is the total latency and we even allow players to have weights. The main theorem of this subsection was first proved in [2] for mixed Nash equilibria. Here we generalize it to correlated equilibria. Our proof is shorter and in our opinion simpler, but it borrows a lot of ideas from [2]. We will need the following lemma:

Lemma 3. For every non negative real $\alpha, \beta$, it holds

$$
\alpha \beta+\beta^{2} \leq \frac{\sqrt{5}-1}{4} \alpha^{2}+\frac{\sqrt{5}+5}{4} \beta^{2}
$$

Proof. We want to find $\lambda$ and $k$ that minimize $P A=\frac{\lambda}{k-1}$ and satisfy $\alpha \beta+\beta^{2} \leq \frac{1}{k} \alpha^{2}+\frac{\lambda}{k} \beta^{2}$. We see that this is achieved for $k=1+\sqrt{5}$ and $\lambda=(3 \sqrt{5}+5) / 2$.

Theorem 6. For linear weighted congestion games, the correlated price of anarchy of the total latency is at most $\frac{3+\sqrt{5}}{2} \approx 2.618$.

Proof. Let $q$ be a correlated equilibrium and $P$ be an optimal (or any other) allocation. The cost of player $i$ at the correlated equilibrium is $c_{i}(q)=\sum_{s \in \mathcal{S}} q(s) c_{i}(s)=\sum_{s \in \mathcal{S}} q(s) \sum_{e \in s_{i}} f_{e}\left(\theta_{e}(s)\right)$, where $\theta_{e}(s)$ is the total load on the facility $e$ for the allocation $s$. We want to bound the expected total latency: $C(q)=E\left[\sum_{e \in E} l_{e} f_{e}\left(l_{e}\right)\right]=\sum_{s \in \mathcal{S}} q(s) \sum_{e \in E} \theta_{e}(s) f_{e}\left(\theta_{e}(s)\right)$, where $l_{e}$ is a random variable indicating the actual load on the facility $e$, with respect to the optimal cost $C(P)=\sum_{e \in E} \theta_{e}(P) f_{e}\left(\theta_{e}(P)\right)$.

At the correlated equilibrium

$$
c_{i}(q)=\sum_{s \in \mathcal{S}} q(s) \sum_{e \in s_{i}} f_{e}\left(\theta_{e}(s)\right) \leq \sum_{s \in \mathcal{S}} q(s) \sum_{e \in P_{i}} f_{e}\left(\theta_{e}\left(s_{-i}, P_{i}\right)\right) \leq \sum_{s \in \mathcal{S}} q(s) \sum_{e \in P_{i}} f_{e}\left(\theta_{e}(s)+w_{i}\right)
$$

where $\left(s_{-i}, P_{i}\right)$ is the usual notation in Game Theory to denote the allocation that results when we replace $s_{i}$ by $P_{i}$.

If we multiply this inequality with $w_{i}$ we get 


$$
\sum_{s \in \mathcal{S}} q(s) \sum_{e \in s_{i}} f_{e}\left(\theta_{e}(s)\right) w_{i} \leq \sum_{s \in \mathcal{S}} q(s) \sum_{e \in P_{i}} f_{e}\left(\theta_{e}(s)+w_{i}\right) w_{i}
$$

If we sum over all players $i$, we can bound the expected total latency as

$$
\begin{array}{r}
\sum_{i \in N} \sum_{s \in \mathcal{S}} q(s) \sum_{e \in s_{i}} f_{e}\left(\theta_{e}(s)\right) w_{i}=\sum_{s \in \mathcal{S}} q(s) \sum_{e \in E} \theta_{e}(s) f_{e}\left(\theta_{e}(s)\right)=C(q) \leq \\
\sum_{i \in N} \sum_{s \in \mathcal{S}} q(s) \sum_{e \in P_{i}} f_{e}\left(\theta_{e}(s)+w_{i}\right) w_{i}=\sum_{i \in N} \sum_{s \in \mathcal{S}} q(s) \sum_{e \in P_{i}}\left(a_{e}\left(\theta_{e}(s)+w_{i}\right)+b_{e}\right) w_{i} \leq \\
\sum_{s \in \mathcal{S}} q(s)\left(\sum_{e \in E} a_{e}\left(\theta_{e}(s) \theta_{e}(P)+\theta_{e}^{2}(P)\right)+\sum_{e \in E} b_{e} \theta_{e}(P) \leq\right. \\
\sum_{s \in \mathcal{S}} q(s)\left(\sum_{e \in E} a_{e}\left(\frac{\sqrt{5}-1}{4} \theta_{e}^{2}(s)+\frac{\sqrt{5}+5}{4} \theta_{e}^{2}(P)\right)+\sum_{e \in E} b_{e} \theta_{e}(P) \leq\right. \\
\frac{\sqrt{5}-1}{4} C(q)+\frac{\sqrt{5}+5}{4} C(P),
\end{array}
$$

and the theorem follows.

\section{Conclusion and open problems}

We considered the price of anarchy and stability of correlated equilibria for linear congestion games. Some of our results extend directly to polynomial cost functions but we leave the details for the full version of the paper.

Our results point directly to interesting open questions such as to close the gap between the lower bound of 1.577 and the upper bound of 1.6 of the price of stability (Theorems 1 and 2). We also left open some cases about the maximum cost or symmetric games.

\section{References}

[1] R. Aumann. Subjectivity and correlation in randomized games. Journal of Mathematical Economics,1, pages 67-96, 1974.

[2] B. Awerbuch, Y. Azar and A. Epstein. The Price of Routing Unsplittable Flow. 37th Annual ACM Symposium on Theory of Computing (STOC), 2005.

[3] E. Anshelevich, A. Dasgupta, J. Kleinberg, E. Tardos, T. Wexler and T. Roughgarden. The Price of Stability for Network Design with Fair Cost Allocation. In 45th Annual IEEE Symposium on Foundations of Computer Science (FOCS), pages 59-73, 2004.

[4] G. Christodoulou and E. Koutsoupias. The price of anarchy of finite congestion games. 37th Annual ACM Symposium on Theory of Computing (STOC), 2005. http://www.di.uoa.gr/〜elias/publications/paper-ck05.html

[5] J. R. Correa, A. S. Schulz and N. S. Moses. Computational Complexity, Fairness, and the Price of Anarchy of the Maximum Latency Problem. In Proceedings of the 10th International Conference on Integer Programming and Combinatorial Optimization (IPCO), pages 59-73, 2004.

[6] A. Czumaj, P. Krysta, B. Vöcking. Selfish traffic allocation for server farms. In Proceedings on 34th Annual ACM Symposium on Theory of Computing (STOC), pages 287-296, 2002. 
[7] A. Czumaj and B. Vöcking. Tight Bounds for Worst-case Equilibria. In Proceedings of the 13th Annual ACM-SIAM Symposium on Discrete Algorithms (SODA), pp. 413-420, January 2002.

[8] A. Fabrikant, C. Papadimitriou, and K. Tulwar. On the complexity of pure equilibria. In Proceedings of the 36th Annual ACM Symposium on Theory Of Computing (STOC), pages 604-612, June 2004.

[9] D. Fotakis, S. C. Kontogiannis and P. G. Spirakis. Selfish Unsplittable Flows. In Proceedings of the 31st International Colloquium on Automata, Languages and Programming (ICALP), pages 593-605, 2004.

[10] M. Gairing, T. Lücking, M. Mavronicolas and B. Monien. The Price of Anarchy for Polynomial Social Cost. In Proceedings of the 29th International Symposium of Mathematical Foundations of Computer Science (MFCS), pages 574-585, 2004.

[11] M. Gairing, T. Lücking, M. Mavronicolas and B. Monien. Computing Nash equilibria for scheduling on restricted parallel links. In Proceedings of the 36th Annual ACM Symposium on Theory of Computing (STOC), pages 613-622, 2004.

[12] M. Gairing, T. Lücking, M. Mavronicolas, B. Monien and M. Rode. Nash Equilibria in Discrete Routing Games with Convex Latency Functions. In Proceedings of the 31st International Colloquium on Automata, Languages and Programming (ICALP), pages 645657, 2004.

[13] E. Koutsoupias, M. Mavronicolas, and P. Spirakis. Approximate Equilibria and Ball Fusion. In Proceedings of the 9th International Colloquium on Structural Information and Communication Complexity (SIROCCO), 2002

[14] E. Koutsoupias and C. H. Papadimitriou. Worst-case equilibria. In Proceedings of the 16th Annual Symposium on Theoretical Aspects of Computer Science, pages 404-413, 1999.

[15] T. Lücking, M. Mavronicolas, B. Monien and M. Rode. A New Model for Selfish Routing. In Proceedings of the 21st Annual Symposium on Theoretical Aspects of Computer Science (STACS), pages 547-558, 2004.

[16] M. Mavronicolas and P. G. Spirakis. The price of selfish routing. In Proceedings on 33rd Annual ACM Symposium on Theory of Computing (STOC), pages 510-519, 2001.

[17] I. Milchtaich. Congestion Games with Player-Specific Payoff Functions. Games and Economic Behavior 13, pages 111-124, 1996.

[18] D. Monderer and L. S. Shapley. Potential Games. Games and and Economic Behavior 14, pages 124-143, 1996.

[19] M. J. Osborne and A. Rubinstein. A Course in Game Theory. The MIT Press, 1994.

[20] C. H. Papadimitriou. Algorithms, games, and the Internet. In Proceedings of the 33rd Annual ACM Symposium on the Theory of Computing, pages 749-753, 2001.

[21] C. H. Papadimitriou. Computing Correlated Equilibria in Multiplayer Games. 37th Annual ACM Symposium on Theory of Computing (STOC), 2005.

[22] C. H. Papadimitriou and T. Roughgarden. Computing Equilibria in Multi-Player Games. In Proceedings of the Sixteenth Annual ACM-SIAM Symposium on Discrete Algorithms (SODA), pages 82-91, 2005. 
[23] R. W. Rosenthal. A class of games possessing pure-strategy Nash equilibria. International Journal of Game Theory, 2:65-67, 1973.

[24] T. Roughgarden. The price of anarchy is independent of the network topology. In Proceedings of the 34th Annual ACM Symposium on the Theory of Computing, pages 428-437, 2002. Journal of Computer and System Sciences, 67(2):341-364, September 2003.

[25] T. Roughgarden. The maximum latency of selfish routing. In Proceedings of the Fifteenth Annual ACM-SIAM Symposium on Discrete Algorithms (SODA), pages 980-981, 2004.

[26] T. Roughgarden and E. Tardos. How bad is selfish routing? Journal of the ACM, 49(2):236259, 2002.

[27] T. Roughgarden and E. Tardos. Bounding the inefficiency of equilibria in nonatomic congestion games. Games and Economic Behavior, 47(2):389-403, 2004.

[28] S. Suri, C. D. Tóth and Y. Zhou. Selfish load balancing and atomic congestion games. In Proceedings of the sixteenth annual ACM symposium on Parallelism in algorithms and architectures, pages 188-195, 2004. 